\title{
Evaluating the effect of the English Enhanced Recovery Partnership Programme: A national analysis of length of stay from 2009-2015
}

International Forum on Quality and Safety in Healthcare, 12-15 April 2016, Gothenburg, Sweden.

\section{Context}

In April 2009 the Department of Health (DOH) launched the Enhanced Recovery Partnership Programme (ERPP), a 2-year national improvement programme, to promote the uptake of Enhanced Recovery after Surgery (ERAS) within the National Health Service (NHS).

\section{Problem}

The ERPP was launched to support the NHS to implement and realise the benefits of enhanced recovery, across all hospitals and surgical procedures involving the colorectal, urology, gynaecology, and musculoskeletal specialties. This paper analysis the effect on LOS of the ERPP for the 4 years after it finished in March 2011.

Prior to the ERPP in 2009 the adoption of ERAS was fragmented across the NHS, limited to pioneers and enthusiasts. The best assessment at this time was that around $40-50$ providers had clinical teams that practised enhanced recovery in one or more specialty, but there was no comprehensive assessment available of the rigour with which these teams had implemented the clinical elements of the pathway. Many other teams had implemented some of the elements as part of their on-going development of clinical practice

\section{Intervention}

ERAS is a multi-modal approach to care which has been shown to reduce mortality, morbidity and length of stay across a range of surgical procedures. ERAS optimises the perioperative pathway by minimising the surgical stress response by using techniques such as minimally invasive surgery, regional anaesthetic techniques, opioid sparing pain management, nutrition and fluid management and early mobilisation. Central to the enhanced recovery concept is involvement of the patient as a partner in their care.

\section{Strategy for change}

The DOH wanted to introduce ERAS to help reduce and address the wide variations in length of stay (LOS) found across common elective surgical procedures. ERAS pathways were therefore an attractive approach in order to improve clinical, economical and operational outcomes. In year 1 , the ERPP focussed on increasing awareness of ERAS through events, conferences, and producing supportive literature and online resources. In year 2, the ERPP focussed on spread, adoption and sustainability of ERAS and amongst other activities produced a national ERAS database as well as encouraging regional support through the strategic health authorities. Since the end of the programme there has been no on-going $\mathrm{DOH}$ programme.
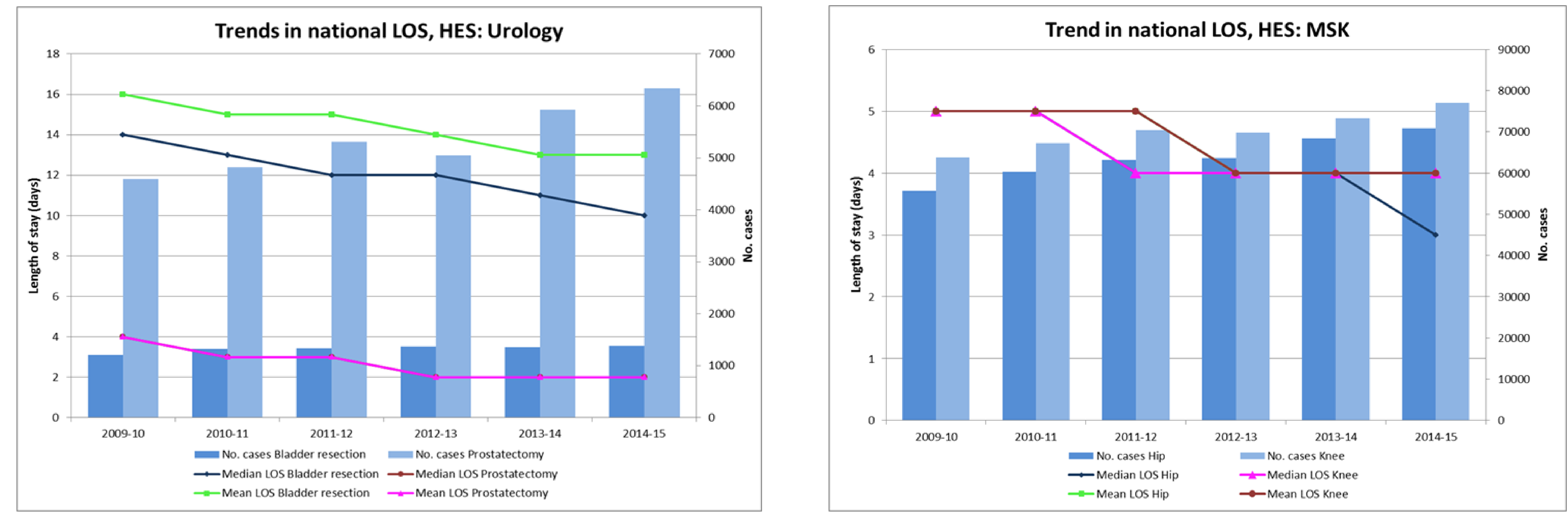

Trend in national LOS, HES: Hysterectomy

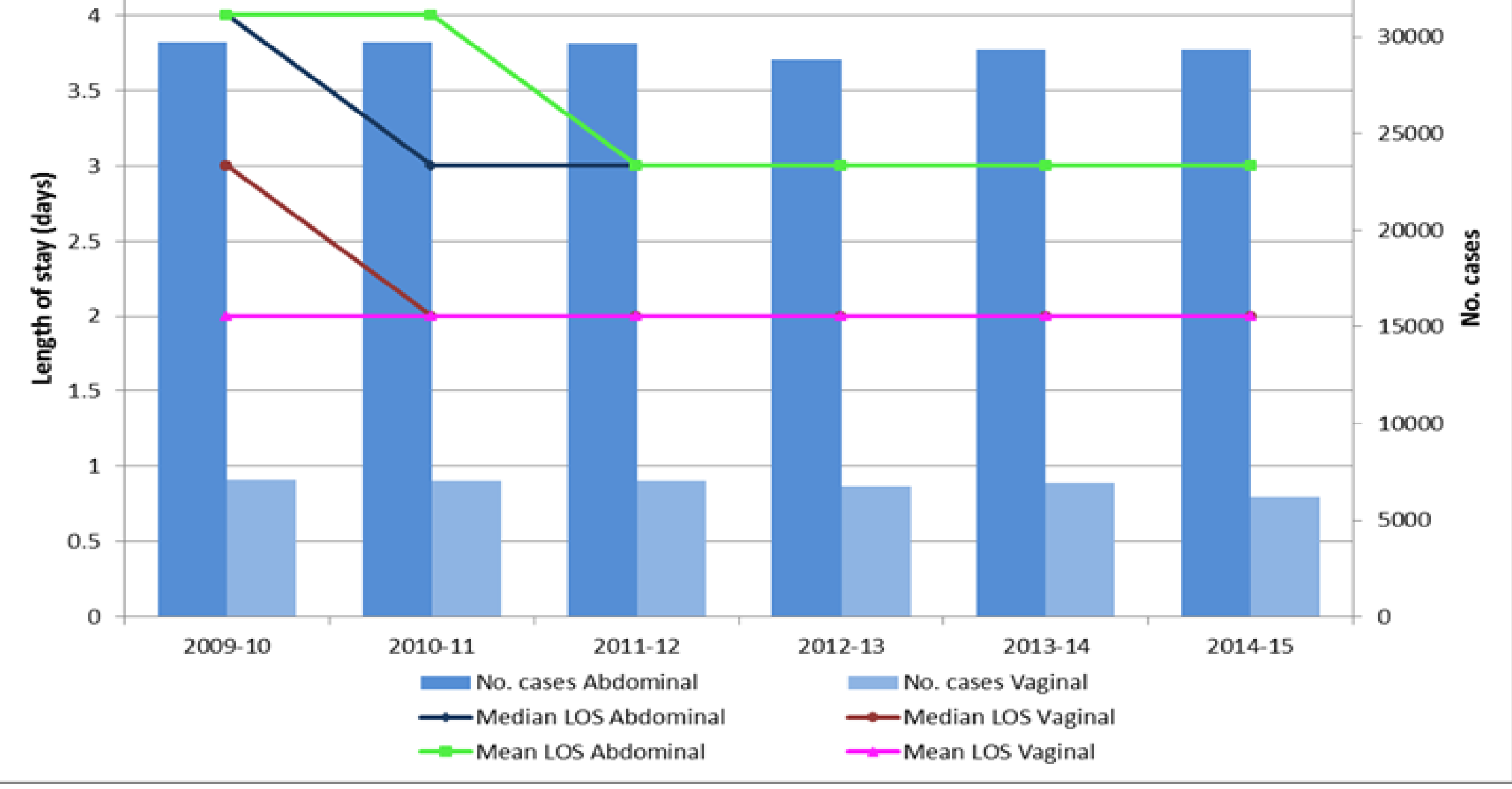

Measurement of improvement

Hospital Episode Statistics (HES) were analysed on data using admission dates from April 2009 to March 2015. The data analysed included a count of spells, mean length of stay (LOS) and median LOS for the 8 ERAS procedure groups split by trust. All analyses were conducted on inpatient elective admissions (excluding day cases) and restricted to spells which have only a single episode. The procedure groups were defined by OPCS 4.7 codes specified by the DOH and consist of the following eight groups: Primary hip replacement, Primary knee replacement, Colectomy, Excision of rectum, abdominal hysterectomy, vaginal hysterectomy, Bladder resection and Prostatectomy.

\section{Effects of changes}

Across England aggregated median LOS has reduced for all surgical procedures. The colorectal procedures (colectomy and excision of rectum) and the gynaecological procedures (Vaginal and abdominal hysterectomy) saw most reduction in LOS whilst the ERPP was active 2009-11. Since 2011/12 only one of these procedures (excision of rectum) has seen a reduction in median LOS. In contrast, the musculoskeletal and urology procedures have seen a continued general reduction in both mean and median LOS.
Trend in national LOS, HES: Colorectal

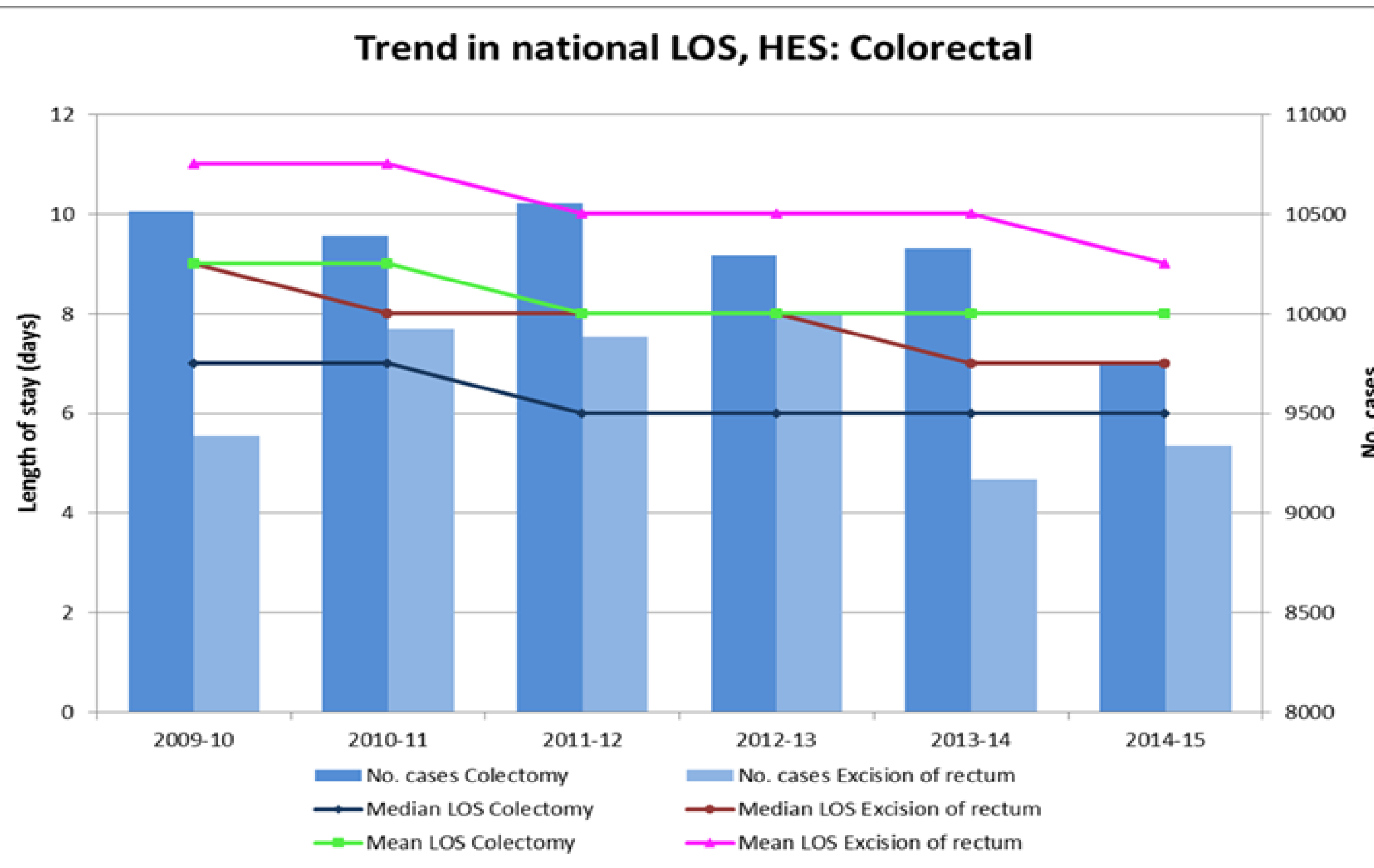

\section{Lessons learnt}

Improvements to LOS following the ERPP have been seen, however improvements have stalled in some specialties, and further improvements are still possible across all specialties when outcomes are compared to international exemplar sites.

\section{Message for others}

National spread, adoption, and sustainability of best practice models of care such as ERAS requires on-going support, facilitation and promotion by national bodies.

\section{Acknowledgements}

The author would like to acknowledge that this work forms part of a larger clinical investigation involving the following members of the ERAS Society UK; Dr D. McDonald, Dr R. Barlow, Dr F. Carter, Dr M. Scott, and Mr N. Francis.

\section{프를 $\mathrm{cec}$ RECOVERY ERAS Society (UK) www.erasuk.net}

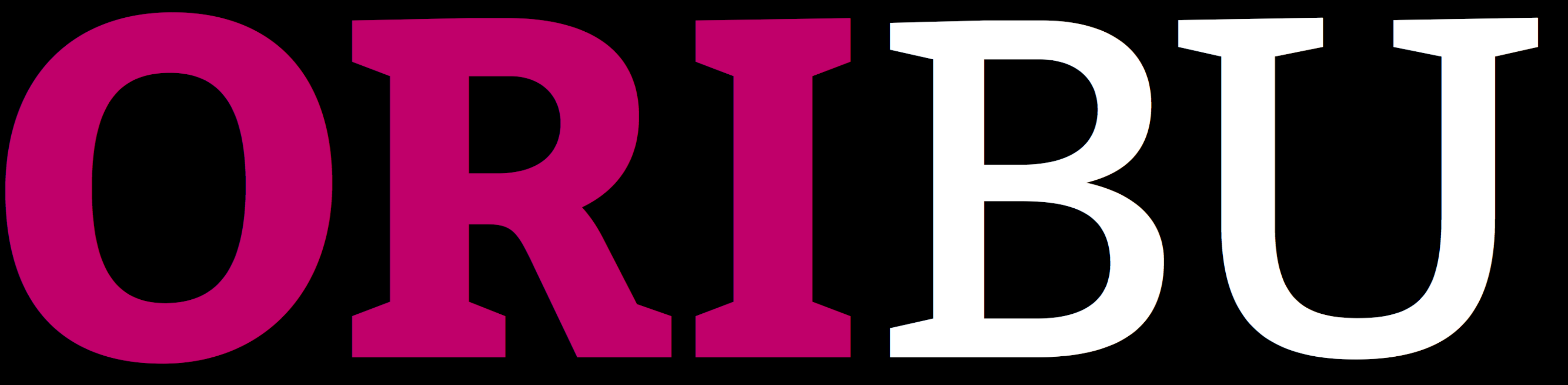

